\title{
Atomic Structure of Through-Thickness Steps in a Grain Boundary
}

\author{
A. Gautam ${ }^{1}$, F. Lançon ${ }^{2}$, V. Radmilovic ${ }^{3}$ and U. Dahmen ${ }^{1}$ \\ 1. National Center for Electron Microscopy, LBNL, Berkeley, CA 94720 USA. \\ ${ }^{2 .}$ Laboratoire de Simulation Atomistique (L Sim), SP2M, INAC, CEA, 38054 Grenoble, France. \\ 3. University of Belgrade, Faculty of Technology and Metallurgy, Nanotechnology and Functional \\ Materials Center, Belgrade, Serbia.
}

Atomic-scale mechanisms of interface motion are of key interest for processes such as grain growth, deformation and phase transformations. For singular interfaces, migration typically involves nucleation and propagation of steps. The atomic structure of such steps can be observed directly in a transmission electron microscope as long as the step is parallel to the electron beam. In any other orientation, steps result in overlapping grains along the direction of projection and therefore cannot be interpreted directly. In this work we combine high resolution transmission electron microscopy (HRTEM) and atomistic modeling techniques to analyze the structure of a through-thickness step in a grain boundary.

The $<110>90^{\circ}$ tilt grain boundary in gold is incommensurate because the repeat periods of the (001) and (110) planes of the two grains that are aligned across the interface are in the ratio of $1: \sqrt{2}$. A previous analysis based on the Frenkel-Kontorova model showed this interface to exhibit superglide behavior $[1,2]$, lending particular interest to the study of its atomic structure and its topological defects such as steps and disconnections [3]. The boundary was imaged using a freestanding bicrystal thin film prepared by physical vapor deposition of high purity gold onto a sacrificial (100) Ge substrate [4]. To study the grain boundary structure, we used the aberration corrected TEAM microscope at the National Center for Electron Microscopy [5]. Figure 1 shows an analysis of a step along the beam direction. The typical appearance in HRTEM images is shown in (a) with the defect area circled and shaded. The same structure in high-angular dark field STEM is shown in (b). A corresponding image simulation (c) reproduces the observed image in (a) very well. The model structure shown in (d) illustrates the 3D nature of the step (lightly shaded) and highlights the atoms of the upper crystal above the step that overlap the lower crystal below the step with arrows.

Figure 2a shows an HRTEM image of a larger segment of such a boundary. The rectangular area marked by a white outline and shading contains several defects of the type shown in Figure 1, indicating a step along the beam direction. The solid line, indicating the position of the boundary clearly shows how the boundary steps toward the upper grain when proceeding from left to right. The dashed line marks the position of the boundary at a different level along the direction of projection, indicating the part of the step that is buried in the thickness of the foil. To illustrate this geometry, a cross-sectional view of the interface is illustrated in (b) by showing a single atomic plane at the core of the boundary in a projection normal to (a). In this view, the two grains are distinguished by their characteristic square and rectangular atomic arrangements. The step is outlined by a white line, again using solid and dashed lines to indicate different levels along the viewing direction. To clarify the geometry, the inset sketch shows the step in perspective view. This boundary was modeled by extracting atomic positions from experimental images, adding the step geometry shown in (b) and relaxing the structure using molecular dynamics simulations for a period of $50 \mathrm{ps}$ at $300 \mathrm{~K}$. The atomic positions shown in (b) represent an average of 50 configurations simulated over a period 50ps. This work shows that a structural understanding of 
through-thickness steps in grain boundaries can be of great importance to our understanding of the mechanisms of interface migration [6].

\section{References:}

[1] F. Lançon, Europhysics Letters 57, 74 (2002).

[2] F. Lancon et al., Nano Lett 10, 695 (2010).

[3] K.H. Westmacott, S. Hinderberger and U. Dahmen, Philosophical Magazine 81, 1547 (2001).

[4] R.C. Pond, D.L. Medlin, A. Serra, Philosophical Magazine 86, 4667 (2006).

[5] U. Dahmen et al., Phil. Trans. Roy. Soc. A 367, 3795 (2009).

[6] This work is supported by the Director, Office of Science, Office of Basic Energy Sciences, of the U.S. Department of Energy under Contract No. DE-ACO3-76SFOOO98, and by the Direction des Sciences de la Matière of the CEA.
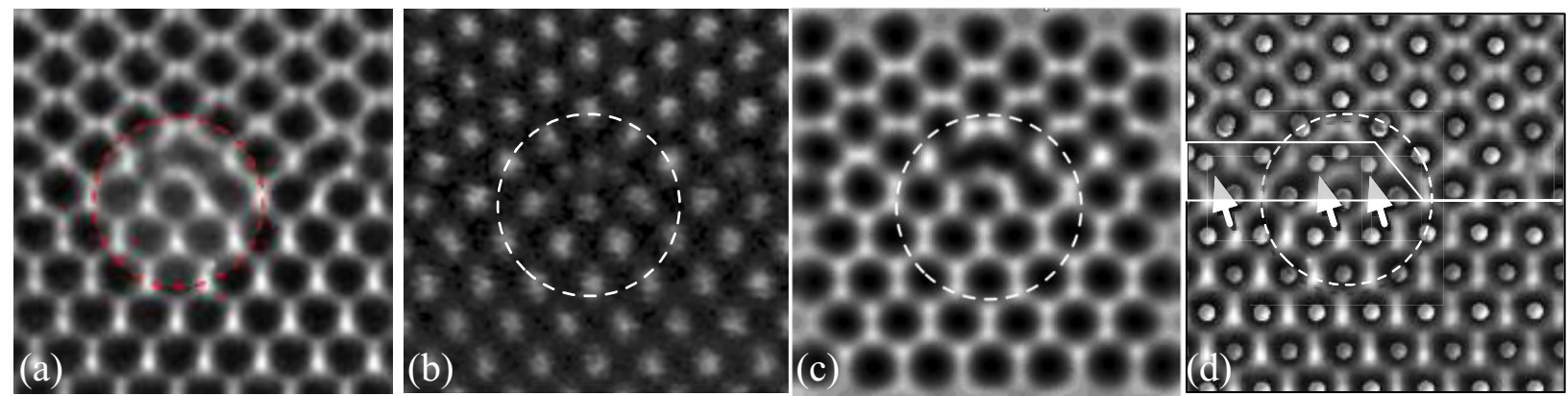

Figure 1, Typical through-thickness defect in the incommensurate 110/001 grain boundary in $90^{\circ}$ $<110>$ mazed bicrystal of Au. (a) HRTEM image showing defect framed by shaded circle; (b) STEM image showing the same defect; (c) image simulation reproducing the contrast observed in (a); (d) model of buried step showing overlapping crystals in shaded area, with overlapping atoms highlighted by arrows.
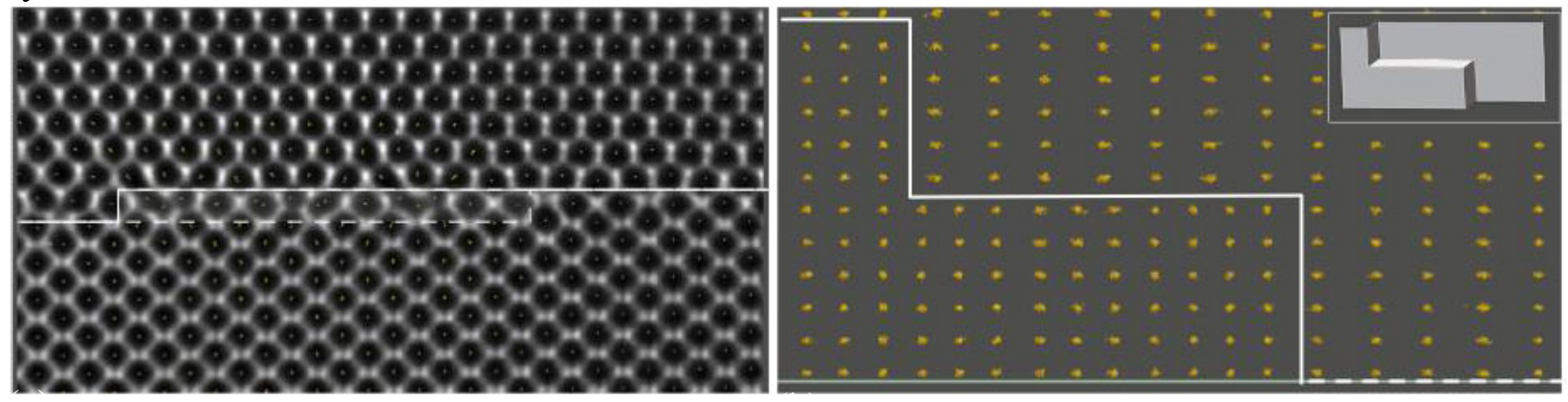

Figure 2. Grain boundary containing a buried step. (a) HRTEM image viewing interface edge-on. The shaded box between lines indicates the length the step. Dashed and solid lines indicate different levels in projection. (b) cross sectional view of the model structure viewed perpendicular to (a) showing only the atomic plane at the core of the boundary containing the step. The white dots represent clusters of overlaid atomic positions after every 1 ps of the 50 ps MD simulation. Inset sketch shows a perspective view of the topology to illustrate the $3 \mathrm{D}$ nature of the step. 